\title{
AN EXISTENCE RESULT FOR A MULTIPOINT BOUNDARY VALUE PROBLEM ON A TIME SCALE
}

\author{
BASANT KARNA AND BONITA A. LAWRENCE
}

Received 31 January 2006; Revised 15 April 2006; Accepted 19 April 2006

We will expand the scope of application of a fixed point theorem due to Krasnosel'skiı and Zabreiko to the family of second-order dynamic equations described by $u^{\Delta \Delta}(t)=$ $f\left(u^{\sigma}(t)\right), t \in[0,1] \cap \mathbb{T}$, with multipoint boundary conditions $u(0)=0, u\left(\sigma^{2}(1)\right)=$ $\sum_{i=1}^{n} \alpha_{i} u\left(\eta_{i}\right)$, and $\sum_{i=1}^{n} \alpha_{i} \leq 1$ for the purpose of establishing existence results. We will determine sufficient conditions on our function $f$ such that the assumptions of the fixed point theorem are satisfied, which in return gives us the existence of solutions.

Copyright (c) 2006 B. Karna and B. A. Lawrence. This is an open access article distributed under the Creative Commons Attribution License, which permits unrestricted use, distribution, and reproduction in any medium, provided the original work is properly cited.

\section{Introduction}

Consider a very general family of dynamic equations of the form

$$
u^{\Delta \Delta}(t)=f\left(u^{\sigma}(t)\right), \quad t \in[0,1] \cap \mathbb{T}
$$

a second-order dynamic equation defined on a time scale, $\mathbb{T}$, that is a subset (closed by definition) of the interval $[0,1]$. (For more information on time scales calculus, see the books of Bohner and Peterson [4, 5] and Hilger [12].) Assume that you know the behavior of the solution at an initial value as well as the relationship between the values of the solution at several other points. In particular, let us consider boundary conditions

$$
u(0)=0, \quad u\left(\sigma^{2}(1)\right)=\sum_{i=1}^{n} \alpha_{i} u\left(\eta_{i}\right), \quad \sum_{i=1}^{n} \alpha_{i} \leq 1,
$$

where $\eta_{i} \in[0,1] \cap \mathbb{T}$. A standard method for establishing the existence of solutions for such dynamic boundary value problems is the application of a fixed point theorem. Many 
such theorems such as Guo-Krasnosel'skiı found in [8], Leggett-Williams [15], and, more recently, Avery-Henderson [3] have been used to develop the various existence results currently available to us. The particular fixed point theorem used determines the corresponding assumptions on the function $f$ in (1.1) that will insure the existence. For example, using the fixed point theorems described above, the existence of positive solutions for (1.1) with various types of boundary conditions has been established in the case when $f>0$. (See Anderson $[1,2]$ and Erbe and Peterson $[6,7]$.)

Sun and $\mathrm{Li}$ [16] took a different track that allowed the function $f$ in (1.1) to change sign. The basis of their proof was a fixed point theorem due to Krasnosel'skil and Zabreiko and they assumed right focal boundary conditions. In [10], Henderson expanded their result to mixed boundary conditions

$$
\begin{gathered}
\alpha u(0)-\beta u^{\Delta}(0)=0, \\
\gamma u\left(\sigma^{2}(1)\right)+\delta u^{\Delta}(\sigma(1))=0,
\end{gathered}
$$

for $\alpha, \beta, \gamma, \delta>0$, and

$$
d=\gamma \beta+\alpha \delta+\alpha \gamma \sigma^{2}(1)>0
$$

In [11] Henderson and Lawrence investigated higher even-ordered problems with SturmLiouville boundary conditions using this result of Krasnosel'skil and Zabreiko. In this paper, we return to the second-order problem and allow for multiple boundary points. First, in Section 2 we lay the foundation for the development of our theorem by stating the fixed point theorem and analyzing its required elements. In Section 3, we determine what the assumptions of the fixed point theorem imply about our dynamic equation and complete the paper with a statement of our existence theorem in Section 4.

\section{Foundational results}

Our main result is an application of the fixed point theorem due to Krasnosel'skil and Zabreiko that follows in its original form. The assumptions of this theorem include two operators; one completely continuous and asymptotically linear and one bounded and linear, and a Banach space to work in. For our particular application, the operators are defined in terms of an appropriate Green's function.

THeorem 2.1. Let E be a Banach space and F a completely continuous operator defined on all of $E$ which is asymptotically linear. If 1 is not an eigenvalue of the operator $F^{\prime}(\infty)$, then $x=$ Fx has at least one solution.

The notation $F^{\prime}(\infty)$, the derivative of $F$ at infinity, denotes a linear operator $A$ that satisfies

$$
\lim _{\|x\| \rightarrow \infty} \frac{\|F x-A x\|}{\|x\|}=0
$$


If such an operator $A$ exists, the operator $F$ is called asymptotically linear. The basis of the proof of this far-reaching theorem (found in [13]) is the following. With no eigenvalue of $1, x-F(x)$ and $x-A(x)$ are of the same homotopy class and therefore have the same index.

Our Banach space of choice is $(X,\|\cdot\|)$, where $X=C\left[0, \sigma^{2}(1)\right]$ and

$$
\|u\|=\sup \left\{|u(t)| t \in\left[0, \sigma^{2}(1)\right]\right\} .
$$

We will construct two required operators using Green's function for the homogeneous equation

$$
-u^{\Delta \Delta}(t)=0, \quad t \in[0,1] \cap \mathbb{T},
$$

with boundary conditions (1.2). This requires the construction of a general Green's function for (2.3) with $n+2$ boundary points that satisfy the conditions laid out in (1.2). To clean up the notation a bit, define $\alpha$ and $T$ as

$$
\begin{gathered}
\alpha \equiv 1-\sum_{i=1}^{n} \alpha_{i} \\
T \equiv \sigma^{2}(1)-\sum_{i=1}^{n} \alpha_{i} \eta_{i} .
\end{gathered}
$$

Green's function is piecewise defined as follows:

(i) for $t \leq s$ and $0 \leq s \leq \eta_{1}$,

$$
G(t, s)=t\left[1-\frac{\sigma(s) \alpha}{T}\right]
$$

and for $\sigma(s) \leq t$ and $0 \leq s \leq \eta_{1}$,

$$
G(t, s)=\sigma(s)\left[1-\frac{t \alpha}{T}\right] ;
$$

(ii) for $t \leq s$ and $\sigma\left(\eta_{1}\right) \leq s \leq \eta_{2}$,

$$
G(t, s)=t\left[1+\frac{1}{T}\left[\sum_{i=1}^{1} \alpha_{i}\left(\sigma\left(\eta_{i}\right)-\sigma(s)\right)-\sigma(s) \alpha\right]\right],
$$

and for $\sigma(s) \leq t$ and $\sigma\left(\eta_{1}\right) \leq s \leq \eta_{2}$,

$$
G(t, s)=\sigma(s)+\frac{t}{T}\left[\sum_{i=1}^{1} \alpha_{i}\left(\sigma\left(\eta_{i}\right)-\sigma(s)\right)-\sigma(s) \alpha\right]
$$

(iii) for $t \leq s$ and $\sigma\left(\eta_{2}\right) \leq s \leq \eta_{3}$,

$$
G(t, s)=t\left[1+\frac{1}{T}\left[\sum_{i=1}^{2} \alpha_{i}\left(\sigma\left(\eta_{i}\right)-\sigma(s)\right)-\sigma(s) \alpha\right]\right],
$$


and for $\sigma(s) \leq t$ and $\sigma\left(\eta_{2}\right) \leq \eta_{3}$,

$$
G(t, s)=\sigma(s)+\frac{t}{T}\left[\sum_{i=1}^{2} \alpha_{i}\left(\sigma\left(\eta_{i}\right)-\sigma(s)\right)-\sigma(s) \alpha\right]
$$

(iv) and finally, for $t \leq s$ and $\sigma\left(\eta_{n}\right) \leq s \leq \sigma^{2}(1) \equiv \eta_{n+1}$,

$$
G(t, s)=\frac{t}{T}\left[\sigma^{2}(1)-\sigma(s)\right]
$$

and for $\sigma(s) \leq t$ and $\sigma\left(\eta_{n}\right) \leq s \leq \sigma^{2}(1) \equiv \eta_{n+1}$,

$$
G(t, s)=\sigma(s)+t\left[\frac{\sigma^{2}-\sigma(s)}{T}-1\right]
$$

In general, this Green's function can be written in closed form as follows:

$$
G(t, s)= \begin{cases}t\left[1+\frac{1}{T}\left[\sum_{i=1}^{k} \alpha_{i}\left(\sigma\left(\eta_{i}\right)-\sigma(s)\right)-\sigma(s) \alpha\right]\right] & \text { if } t \leq s, \sigma\left(\eta_{k}\right) \leq s \leq \eta_{k+1}, \\ \sigma(s)+\frac{t}{T}\left[\sum_{i=1}^{k} \alpha_{i}\left(\sigma\left(\eta_{i}\right)-\sigma(s)\right)-\sigma(s) \alpha\right] & \text { if } \sigma(s) \leq t, \sigma\left(\eta_{k}\right) \leq s \leq \eta_{k+1},\end{cases}
$$

where $k=0,1, \ldots, n, \eta_{0}=0, \eta_{n+1}=\sigma^{2}(1)$, and for $k=0, \sum_{i=1}^{k} \alpha_{i}\left(\sigma\left(\eta_{i}\right)-\sigma(s)\right)=0$.

Note that from the assumptions we have made on the boundary points and the definition of $G(t, s)$ above we have $G(t, s)>0$ for $(t, s) \in\left(0, \sigma^{2}(1)\right) \times(0, \sigma(1))$.

With this Green's function in mind, we define our completely continuous operator, $F: X \rightarrow X:$

$$
(F u)(t)=\int_{0}^{\sigma(1)} G(t, s) f\left(u^{\sigma}\right) \Delta s
$$

The choice is made because it is well known that $u$ is a fixed point of $F$ if and only if $u$ is a solution of (1.1), (1.2). Next consider the linear dynamic equation

$$
u^{\Delta \Delta}(t)+m u(\sigma(t))=0, \quad t \in[0,1] \cap \mathbb{T},
$$

satisfying conditions (1.2). We know that solutions of this multipoint problem are fixed points of the bounded linear operator

$$
(A u)(t)=m \int_{0}^{\sigma(1)} G(t, s) u(\sigma(s)) \Delta s,
$$

where $G(t, s)$ is Green's function for (2.3) satisfying (1.2). Hence, this operator is the operator $A$ required by the fixed point theorem. 


\section{A motivation of the main result}

With the operators $F$ and $A$ in mind, we must first verify that 1 is not an eigenvalue for the operator $A$. In the process we will discover the nature of our constant $m$ in (2.15). In the trivial case, when $m=0$, the only solution of (2.15), (1.2) is trivial and therefore $A$ does not have 1 for an eigenvalue. In the event that $m \neq 0$ and using the definition of the operator and properties of inequalities we have the following estimate:

$$
\begin{aligned}
\|A u\| & =\sup _{t \in[0,1] \cap \mathbb{T}}\left|m \int_{0}^{\sigma(1)} G(t, s) u^{\sigma}(s) \Delta s\right| \\
& =|m| \sup _{t \in[0,1] \cap \mathbb{T}}\left|\int_{0}^{\sigma(1)} G(t, s) u^{\sigma}(s) \Delta s\right| \\
& \leq|m| \sup _{t \in[0,1] \cap \mathbb{T}} \int_{0}^{\sigma(1)} G(t, s)\left|u^{\sigma}(s)\right| \Delta s \\
& \leq|m|\|u\| \sup _{t \in[0,1] \cap \mathbb{T}} \int_{0}^{\sigma(1)} G(t, s) \Delta s .
\end{aligned}
$$

Now if we choose $m$ such that

$$
m<b=\frac{1}{\sup _{t \in[0,1] \cap \mathbb{\pi}} \int_{0}^{\sigma(1)} G(t, s) \Delta s},
$$

then the above expressions reduce to

$$
\|A u\|<b\|u\| \frac{1}{b}=\|u\|
$$

and 1 is not an eigenvalue of $A$.

The next important question we need to answer is the following: what is needed to insure that the limit in the assumptions of the fixed point theorem, that is,

$$
\lim _{\|x\| \rightarrow \infty} \frac{\|F x-A x\|}{\|x\|}=0
$$

will be satisfied? Consider first the numerator of this expression, and utilizing the nature of $G$ we have

$$
\begin{aligned}
\|F(u)-A(u)\| & =\sup _{t \in[0,1] \cap \mathbb{\mathbb { T }}}\left|m \int_{0}^{\sigma(1)} G(t, s)\left[f\left(u^{\sigma}(s)\right)-m u^{\sigma}(s)\right] \Delta s\right| \\
& \leq \sup _{t \in[0,1] \cap \mathbb{\mathbb { T }}} m \int_{0}^{\sigma(1)} G(t, s)\left|f\left(u^{\sigma}(s)\right)-m u^{\sigma}(s)\right| \Delta s .
\end{aligned}
$$

We need to show that we can bound this last expression by

$$
\varepsilon \cdot\|u\| \cdot K, \quad \text { where } K \text { is a positive constant, }
$$


for any $\varepsilon>0$. One method for arriving at this end would be to show that

$$
\left|f\left(u^{\sigma}(s)\right)-m u^{\sigma}(s)\right|<\varepsilon\|u\| .
$$

We make the assumption that our function $f$ in (1.1) satisfies the following limit:

$$
\lim _{r \rightarrow \infty} \frac{f(r)}{r}=m
$$

where $m$ is defined in (3.2). Choose any $\varepsilon>0$. The proceeding limit insures us that we can find a constant $C_{1}>0$ such that when $\|r\|>C_{1}$,

$$
|f(r)-m r|<\varepsilon|r|
$$

Using this constant $C_{1}$, we calculate another constant $C$ defined as

$$
C=\sup _{|r| \leq C_{1}}|f(r)|
$$

and note that for $u^{\sigma}(s) \leq C_{1}$ and for $s \in[0, \sigma(1)] \cap \mathbb{T}$,

$$
\left|f\left(u^{\sigma}(s)\right)-m u^{\sigma}(s)\right| \leq\left|f\left(u^{\sigma}(s)\right)\right|+|m|\left|u^{\sigma}(s)\right| \leq C+|m| C_{1} .
$$

Also, we can find $M>C_{1}$ large enough so that

$$
C+|m| C_{1}<M \cdot \varepsilon,
$$

and therefore, if we choose $u \in X$ such that $\|u\|>M$ our expression (3.11) reduces to

$$
\left|f\left(u^{\sigma}(s)\right)-m u^{\sigma}(s)\right| \leq \varepsilon M \leq \varepsilon\|u\| .
$$

For the case when $u^{\sigma}(s)>C_{1}$, condition (3.8) gives us the same bound

$$
\left|f\left(u^{\sigma}(s)\right)-m u^{\sigma}(s)\right| \leq \varepsilon\left|u^{\sigma}(s)\right| \leq \varepsilon\|u\| .
$$

So, for all $u$ such that $\|u\|>M$, we have the desired bound for the norm of the difference of $F$ and $A$,

$$
\begin{aligned}
\|F(u)-A(u)\| & =\sup _{t \in[0,1] \cap \mathbb{\pi}}\left|m \int_{0}^{\sigma(1)} G(t, s)\left[f\left(u^{\sigma}(s)\right)-m u^{\sigma}(s)\right] \Delta s\right| \\
& \leq \varepsilon\|u\| \sup _{t \in[0,1] \cap \mathbb{\pi}} m \int_{0}^{\sigma(1)} G(t, s) \Delta s \\
& <\varepsilon\|u\| \frac{1}{b}
\end{aligned}
$$

or equivalently, our limit holds:

$$
\lim _{\|x\| \rightarrow \infty} \frac{\|F x-A x\|}{\|x\|}=0
$$

We now have created the requisite operators and determined conditions on $f$ in (3.8) that will insure that the prescribed limit holds. Next we have, the existence theorem. 


\section{Existence theorem}

The motivation in Section 3 gives rise to the our main result.

Theorem 4.1. Let $f: \mathbb{R} \rightarrow \mathbb{R}$ be a continuous function such that

$$
\lim _{u \rightarrow \infty} \frac{f(u)}{u}=m
$$

If

$$
\frac{1}{\sup _{t \in[0,1] \cap \mathbb{\pi}} \int_{0}^{\sigma(1)} G(t, s) \Delta s}=b>|m|,
$$

then the nonlinear boundary value problem (1.1), (1.2) has a solution.

Proof. Assume that $f$ satisfies condition (4.1) and that the value $m$ satisfies (4.2). For the prescribed operators, $F$ and $A$, use the operator defined in (2.14) and (2.16), respectively. In Section 3 we verified that $A$ does not have 1 as an eigenvalue and that, with the conditions on $f$, the limit

$$
\lim _{\|x\| \rightarrow \infty} \frac{\|F x-A x\|}{\|x\|}=0
$$

holds. The theorem of Krasnosel'skir and Zabreiko tells us that $F$ has a fixed point, that is,

$$
y(t)=\int_{0}^{\sigma(1)} G(t, s) f\left(y^{\sigma}(s)\right) \Delta s
$$

is a solution of (1.1), (1.2).

The use of this fixed point offers us the opportunity to consider functions that change sign. While this expands the class of functions that can be considered, one must keep in mind the additional assumptions on $f$. The crux of the matter is determining the associated Green's function necessary to define the operators. The authors are currently considering even ordered mixed derivatives such as $\nabla \Delta, \Delta \nabla$, and so forth.

\section{References}

[1] D. R. Anderson, Eigenvalue intervals for a second-order mixed-conditions problem on time scales, International Journal of Nonlinear Differential Equations 7 (2002), no. 1-2, 97-104.

[2] __ Eigenvalue intervals for a second-order Sturm-Liouville dynamic equations, to appear in International Journal of Nonlinear Differential Equations.

[3] R. I. Avery and J. Henderson, Two positive fixed points of nonlinear operators on ordered Banach spaces, Communications on Applied Nonlinear Analysis 8 (2001), no. 1, 27-36.

[4] M. Bohner and A. Peterson, Dynamic Equations on Time Scales. An Introduction with Applications, Birkhäuser Boston, Massachusetts, 2001.

[5] M. Bohner and A. Peterson (eds.), Advances in Dynamic Equations on Time Scales, Birkhäuser Boston, Massachusetts, 2003.

[6] L. H. Erbe and A. C. Peterson, Eigenvalue conditions and positive solutions, Journal of Difference Equations and Applications 6 (2000), no. 2, 165-191. 
[7] _ Positive solutions for a nonlinear differential equation on a measure chain, Mathematical and Computer Modelling 32 (2000), no. 5-6, 571-585.

[8] D. J. Guo and V. Lakshmikantham, Nonlinear Problems in Abstract Cones, Notes and Reports in Mathematics in Science and Engineering, vol. 5, Academic Press, Massachusetts, 1988.

[9] J. Henderson, Multiple solutions for 2 mth order Sturm-Liouville boundary value problems on a measure chain, Journal of Difference Equations and Applications 6 (2000), no. 4, 417-429.

[10] Nontrivial solutions to a nonlinear boundary value problem on a time scale, Communications on Applied Nonlinear Analysis 11 (2004), no. 1, 65-71.

[11] J. Henderson and B. A. Lawrence, Existence of solutions for even ordered boundary value problems on a time scale, Proceedings of the International Conference on Difference Equations, Special Functions, and Applications, Munich, 2006.

[12] S. Hilger, Ein Masskettenkalkül mit Anwendung auf Zentrumsmannigfaltigkeiten, Ph.D. thesis, Universität Würzburg, Würzburg, 1988.

[13] M. A. Krasnosel'eskií and P. P. Zabreǐko, Geometrical Methods of Nonlinear Analysis, Fundamental Principles of Mathematical Sciences, vol. 263, Springer, Berlin, 1984.

[14] V. Lakshmikantham, S. Sivasundaram, and B. Kaymakçalan, Dynamic Systems on Measure Chains, Mathematics and Its Applications, vol. 370, Kluwer Academic, Dordrecht, 1996.

[15] R. W. Leggett and L. R. Williams, Multiple positive fixed points of nonlinear operators on ordered Banach spaces, Indiana University Mathematics Journal 28 (1979), no. 4, 673-688.

[16] J.-P. Sun and W.T. Li, A new existence theorem for right focal boundary value problems on a measure chain, Applied Mathematics Letters 18 (2005), no. 1, 41-47.

Basant Karna: Department of Mathematics, Marshall University, Huntington, WV 25755, USA

E-mail address: karna@marshall.edu

Bonita A. Lawrence: Department of Mathematics, Marshall University, Huntington, WV 25755, USA

E-mail address: lawrence@marshall.edu 